\title{
The mechanism of osmosis, a new paradigm
}

Gerald Pollack has discovered and documented a mechanism for osmosis that I think makes a lot of sense. To start of with, I always found osmosis to be "strange". It sticks out, it does not remind me of other processes in physics or chemistry. The explanations for it, that water "wants to even out its concentration", never actually provided any force for it, in my opinion. So I am biased to feeling like there is a missing ingredient around what the actual mechanism for osmosis is.

What Pollack has discovered is incredibly simple and intuitive once you understand it. $\mathrm{He}$ suggests, and has experimentally proven as well, that water forms a different phase when it contacts surfaces. It does so, because the mass of water itself exerts a pressure onto the surface. This pressure forces the water at the surface into a solid phase. But, since the normal solid phase of water has lower density than liquid water, it will compress the solid phase to the point where it has higher density than liquid water. This compression is possible by removing the hydrogen atoms between the molecular ice sheets.

Simple. Very simple. To conform to electrostatic laws, the molecular sheets also shift sideways relative one another, so that positive faces negative, and vice versa. Pollack has done an incredible job explaining this through his books, published articles, and public speeches.

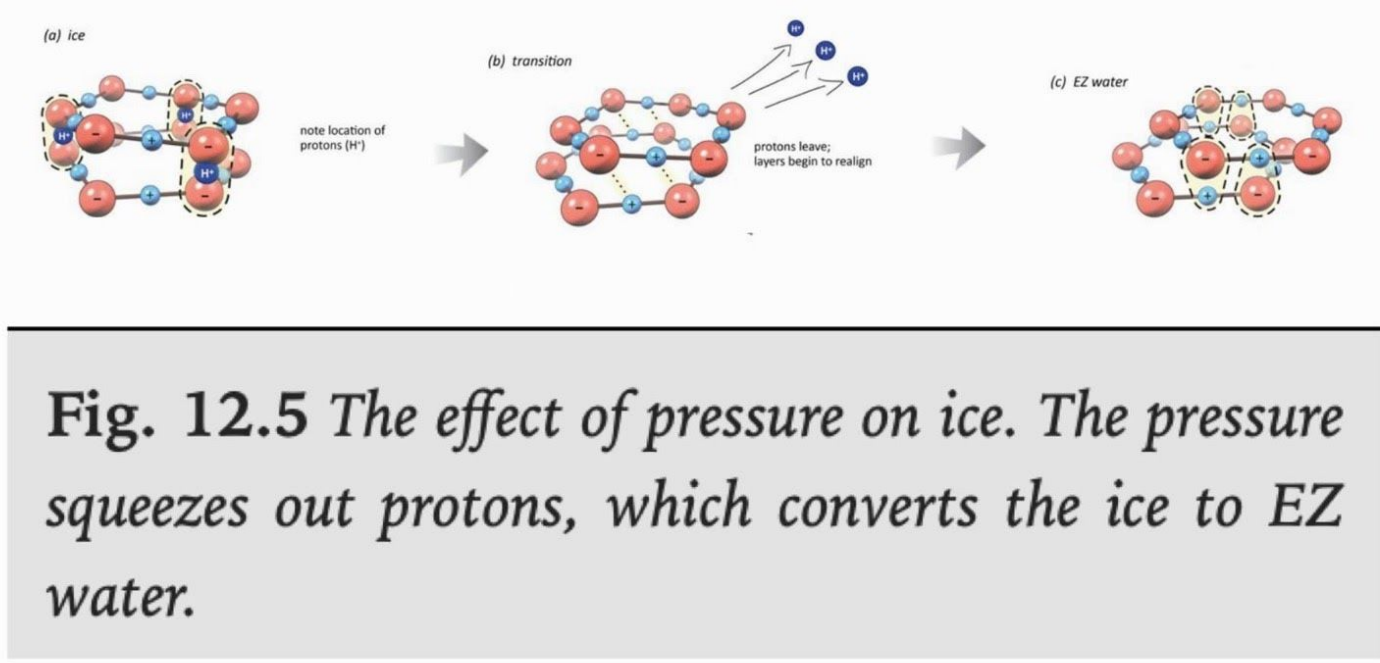

So how does this "dense solid phase" of water cause the phenomena of osmosis? Well, this has to do with that the "surface phase" forms from pressure applied towards the surface. The protons that are ejected from the crystalline structure will be ejected away from the surface, causing a net negative charge at the surface. If the surface was a membrane permeable to protons, these protons would prefer to transfer across the membrane to the other side. They would be "pumped" by the electrostatic charge of the dense solid phase, ultimately powered by the hydrostatic pressure that forms the surface phase, ultimately powered by gravity. 
Hopefully, the understanding that hydrostatic pressure forms an "ionized" solid phase at surfaces that inherently causes the proton half of the phase to want to move over to the other side of the surface, shows a very logical and reasonable explanation for the mechanism of osmosis.

Now, adding water on both sides of a membrane, there will be identical surface phases on both sides. So no proton transfer will happen. But, if the surface phase was diminished on one side, there would still be a net negative charge on that side, and a transfer of protons. This is where solutes come in. A colligative property of added solutes is freezing point depression, the ability for the solid phase to crystalize is impaired by the solutes. The same goes for the "dense solid phase" at surfaces. So adding solutes will impair the surface phase, and cause an electrical gradient, forcing protons to transfer, ultimately powered by the hydrostatic pressure that is caused by gravity.

Gerald Pollack illustrates this all in the image below from his 2013 book The Fourth Phase of Water.

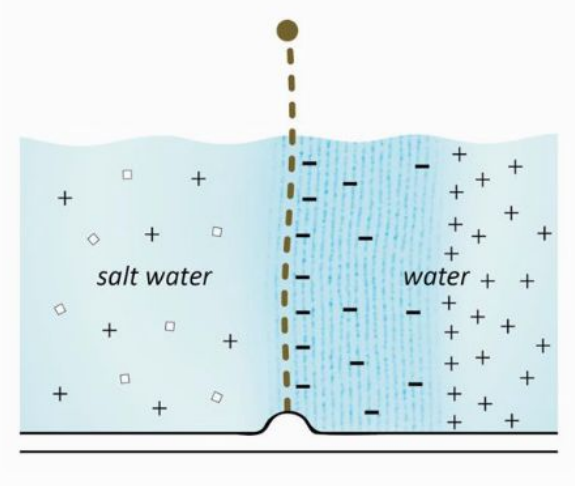

Fig. 11.5 Standard osmosis experiment, with exclusion zones and protons distributed asymmetrically around the dividing membrane.

\section{Exploring this new paradigm further, is there more to it?}

The "dense solid phase" at surfaces provides a mechanism for osmosis, and it explains why adding solutes produces a movement towards the hyperosmotic compartment. This is an incredible discovery, credited to Gerald Pollack, and I am very happy to have learnt about it.

That said, I have considered that there may be more to it. Pollack explains only a movement of protons, and the force behind it is the electrical charge gradient, and the force behind that gradient is the hydrostatic pressure caused by gravity. Why does a transfer of protons specifically cause a transfer of water? 
I think the answer might be that water is actually not moved over the membrane, but, broken down and reconstituted. This assumption ties into ideas I have that redefine what the cell actually is. I see it tie into all of cell biology, and biology and physiology in general. As a theory it is as grandiose as it gets. But it is also incredibly simple, and a very logical assumption.

I think the movement of protons is causing an instability in the dense solid phase at the exosmotic (the compartment water, or protons, moves away from) side of the membrane. The loss of positive charge, and surplus of negative charge, will cause a collapse of the hydroxide ions in the dense solid phase, and a release of electrons.

To understand this it is good to understand the molecular structure of the dense solid phase. Since it has excluded protons between the molecular ice sheets, it has a molecular formula of $(\mathrm{H} 3 \mathrm{O} 2-) \mathrm{n}, \mathrm{H} 2 \mathrm{O}+\mathrm{OH}$ - per hexagon in the lattice, instead of $\mathrm{H} 4 \mathrm{O} 2$ (i.e., $\mathrm{H} 2 \mathrm{O}+\mathrm{H} 2 \mathrm{O}$. ) It is chemically "hydrated hydroxide", $\mathrm{H} 2 \mathrm{O}+\mathrm{OH}$-. See the image below for an illustration of this from Gerald Pollack's The Fourth Phase of Water.

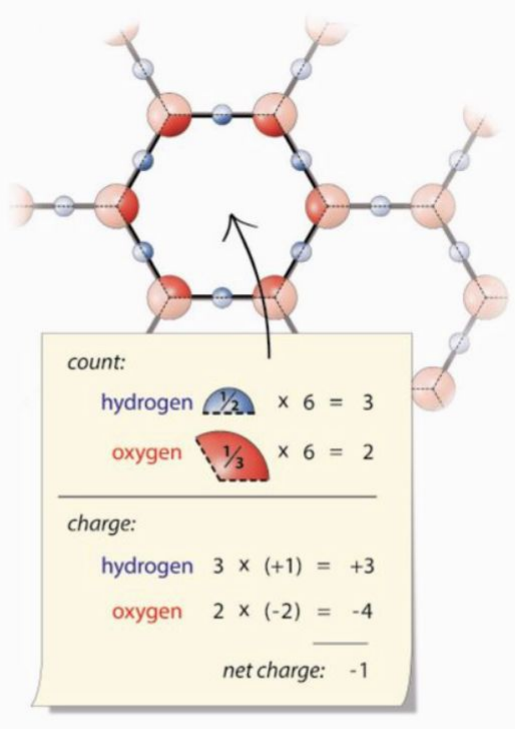

Fig. 4.10 Computation of the net charge of each hexagonal unit. To make the count, represent each atom as a sliceable pie; then count all the pie frac-

There is a well known chemical pathway for the degradation of $\mathrm{OH}-$, that takes place in lots of different well known systems. It also releases electrons. The formula is $4 \mathrm{OH}-\rightarrow \mathrm{O} 2+2$ $\mathrm{H} 2 \mathrm{O}+4 \mathrm{e}-$. My idea is that the loss of positive charge at the exosmotic side, causes $\mathrm{OH}$ - to break down. This leads to the loss of one hydroxide ion per proton transferred, or, one $\mathrm{H} 2 \mathrm{O}$.

Another way to look at this is that the loss of $\mathrm{H}+$ from the exosmotic side favours the oxygen in water to form the dioxide state (oxygen gas) instead of the dihydro state (water), as there is not enough hydrogen for the water state. This is half of the mechanism, and the other half is the charge imbalance. 
Now l'll be clear that I have no indisputable experimental evidence to support this idea, but, as I hope the reader can see, I do have a clear physical and chemical basis in how I derived the idea. I speak for it and promote it because I am curious myself about what actually drives osmosis, and I see potential in this model.

The reaction I described, $4 \mathrm{OH}-\rightarrow \mathrm{O} 2+2 \mathrm{H} 2 \mathrm{O}+4 \mathrm{e}-$, is often called an "oxygen evolution reaction", and it is the basis of an acid-base battery (the source of electricity in an acid-base battery. ) So, it is known to occur, and to consume water. In an acid-base battery, water is likewise produced in the acidic compartment, in a 1:1 ratio. Water "moves".

So back to osmosis. The transfer of protons causes a charge instability at the exosmotic side, as well as a lack of protons (a form of alkalinization), and a breakdown of hydroxide ions with a release of electrons and molecular oxygen. These electrons will flow along the charge gradient, i.e., they follow the proton. So, now the "dense solid phase" has managed to move not just a proton, but also an electron. But how does that move water?

If there is molecular oxygen in the endosmotic compartment, the side water "moves" to in osmosis and so far in this model the side the protons and electrons moved to, then it could combine with the protons and electrons, just like molecular oxygen does in an acid-base battery (causing the production of water in the acidic compartment. ) So, now with that, we have water breaking down on one side, and water being produced on the other. Powered by hydrostatic pressure generating a "surface phase" that inherently wants to transfer protons and electrons.

This electrical circuitry of osmosis is illustrated somewhat in the image below.

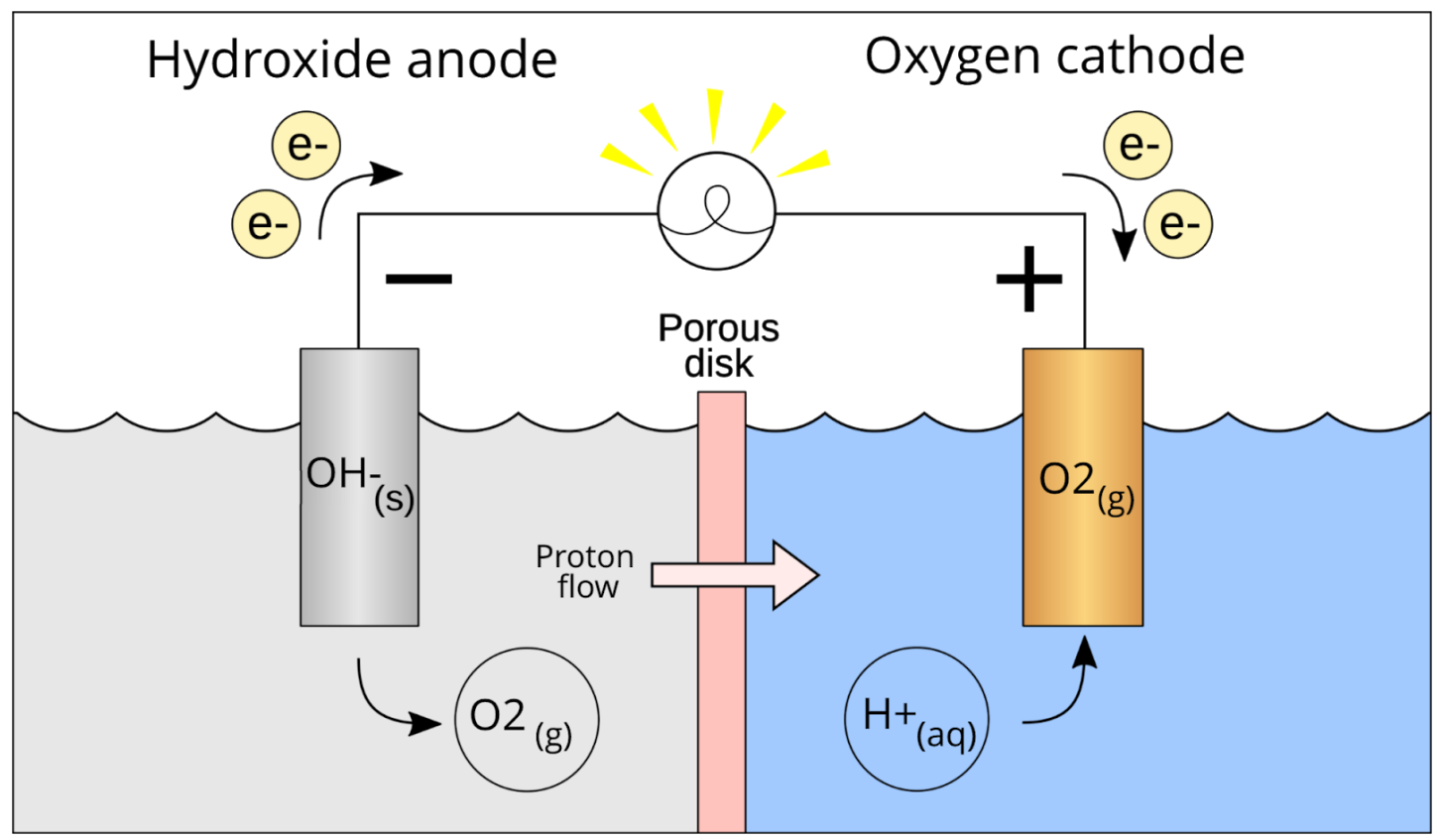

Now consider placing electrically powered machinery within the membrane. I think life might have done so. And, substituted the protons for $\mathrm{Na}+$ and $\mathrm{K}+$, but relying on the same source of electrical discharge, the redox balance between the dihydro and dioxide state of oxygen with the water form "storing" electricity. 
This is not proven by experimental evidence, although it is quite easy to think of experiments that would conclusively prove or disprove the model. It is just based on deductive reasoning from myself, I think the idea seems to have potential, and I believe others may be able to see that potential for themselves, using the basic rules I have described to derive it for themselves, independently of myself.

Also, this "surface phase" also explains surface tension, and is in that case formed from the atmosphere applying pressure.

Sincerely, Johan Nygren 


\section{References}

Pollack, G. H. (2014). Cell electrical properties: reconsidering the origin of the electrical potential. Cell Biology International, 39(3), 237-242. https://doi.org/10.1002/cbin.10382

Zhao, Q., Ovchinnikova, K., Chai, B., Yoo, H., Magula, J., \& Pollack, G. H. (2009). Role of Proton Gradients in the Mechanism of Osmosis. The Journal of Physical Chemistry B, 113(31), 10708-10714. https://doi.org/10.1021/jp9021568

Pollack, G., 2013. The Fourth Phase Of Water. Seattle: Ebner and Sons.

Weng, G.-M., Li, C.-Y. V., \& Chan, K.-Y. (2019). An Acid-Base Battery with Oxygen Electrodes: A Laboratory Demonstration of Electrochemical Power Sources. Journal of Chemical Education, 96(8), 1701-1706. https://doi.org/10.1021/acs.jchemed.8b00901 